\title{
Spectroscopy of interparticle interactions in ionic and molecular liquids: Novel approaches*
}

\author{
Sviatoslav A. Kirillov ${ }^{\ddagger}$ \\ Institute for Technological and Information Innovations, P.O. Box 263, 03134 Kyiv, \\ Ukraine, and Institute of Sorption and Problems of Endoecology, Ukrainian \\ National Academy of Sciences, 13, Gen. Naumov St., 03680 Kyiv, Ukraine
}

\begin{abstract}
The paper deals with several novel approaches to the studies of interactions and dynamics in liquids. A new, flexible time-correlation function is introduced. It has an analytical counterpart in the frequency domain and enables one to fit even badly overlapped vibrational line profiles obtaining dynamical information at the same time. As examples, quantitative description of the interaction potential in molten salts containing halide complexes of zinc and manganese is presented, the dynamic criterion of complex entities in a melt is introduced, and the spectroscopic features of molten alkali and alkaline earth halides are explained in terms of short-lived collision complexes. Finally, the model treatment of "anomalous" noncoincidence effects in liquids is discussed, and the contribution of repulsion and attraction forces resulting in frequency non-coincidences is separated.
\end{abstract}

\section{INTRODUCTION}

Interactions between complex particles in liquids manifest themselves in essential transformation of spectral lines and bands. Vibrational spectroscopy is a powerful tool for studying interparticle interactions in liquids and solutions in great detail; studies of this kind are usually referred to as spectroscopy of interparticle interactions. Distinctive features of spectroscopy of interparticle interactions are as follows. Molecular spectroscopy describes properties of molecules studied in terms of their vibrational transition frequencies and band parameters (widths, intensities, spectral moments, polarization coefficients). Spectroscopy of molecular interactions focuses on the changes of the above parameters (frequency shifts, frequency noncoincidence effects, line broadening, etc.) caused by the interactions of the probe particle with its surrounding in liquid media. The advantage of such an approach lies in the possibility to describe a diversity of different types of interactions as well as dynamical properties of the probe particle, such as reorientation, the transfer of vibrational excitation to other degrees of freedom, and so on.

As compared with molecular liquids, ionic liquids (molten salts) are systems formed by charged particles involved in extremely strong interparticle interactions. This naturally results in quite specific features in vibrational spectra of ionic liquids.

A detailed comparative review of the applications of spectroscopy of molecular interactions to these types of liquids [1] enables one to make the following conclusions.

(i) Vibrational line shifts and broadening in both molecular and ionic liquids are, in principle, of the same nature. However, strong interactions between ionic species give rise to specific contributions to line shifts (ion-dipole, ion-induced dipole) and widths (ion-dipole dissipative).

\footnotetext{
*Lecture presented at the European Molecular Liquids Group (EMLG) Annual Meeting on the Physical Chemistry of Liquids: Novel Approaches to the Structure, Dynamics of Liquids: Experiments, Theories, and Simulation, Rhodes, Greece, 7-15 September 2002. Other presentations are published in this issue, pp. 1-261.

‡E-mail: kir@i.kiev.ua
} 
(ii) In molecular liquids, noncoincidence effects (frequency differences between isotropic and anisotropic Raman and IR spectra) are mainly "normal" and caused mainly by resonant interactions between particles. In ionic liquids, the resonant interactions are, generally speaking, of no value; noncoincidence effects are anomalous and caused by the short-range repulsion.

(iii) No specific differences exist between reorientational dynamics in molecular liquids and molten salts. Moreover, there are striking analogies between both types of liquids in the reduced time scale.

(iv) In molecular liquids, the difference between the vibrational contributions to the IR, isotropic, and anisotropic Raman line contours is most commonly caused by the existence of cross-terms between the "self" and "exchange" parts of vibrational correlation function. In ionic liquids, the same feature arises mainly from the coupling of vibrational and reorientational dephasing due to the ion-dipole interactions.

(v) The major cause of vibrational dephasing in molecular liquids is Gaussian-Markovian frequency modulation (short phase memory). In ionic liquids, all kinds of modulation processes (from nonMarkovian to purely discrete Markovian, from long to completely absent phase memory) are equally possible. Purely discrete Markovian frequency modulation in melts arises due to the ion-dipole interactions between the probe anion and its surrounding.

The aim of this paper is to put under focus recent advances in the field. First, we describe a novel approach to the line profile analysis based on a new, flexible time-correlation function, which has an analytical counterpart in the frequency domain. Using this function one can fit vibrational line profiles obtaining dynamical information at the same time. Numerous applications of this time-correlation function include analysis of line profiles and dynamics of systems with either single or overlapped lines; furthermore, in some cases it appears possible to quantitatively describe the parameters of the interaction potential in a liquid. We consider halide complexes of zinc and manganese in molten alkali halides and prove that the ion-induced dipole interactions are the motive force of vibrational dephasing. Second, we introduce the dynamic criterion of complex entities in a melt, present an analysis of the Raman spectra of molten alkali and alkaline earth halides, and demonstrate that the spectroscopic features of these systems can be explained in terms of short-lived collision complexes. Finally, due to growing interest to the systems with spectroscopic "anomalies" (negative, blue frequency shifts, and negative noncoincidence effects), we recall some principles of the model treatment of these phenomena and describe a method enabling one to separate the contributions of repulsion and attraction forces resulting in frequency non-coincidences.

\section{NOVEL APPROACH TO LINE PROFILE ANALYSIS}

Most information on molecular interactions in liquids and solutions is deduced from spectroscopic studies of molecular dynamics. These studies are usually based on the analysis of vibrational line profiles by means of passage from the frequency domain to the time domain.

Fourier-transforming line profiles, one gets the so-called time-correlation functions. Isotropic Raman line profiles give time-correlation functions of vibrational relaxation (dephasing). Anisotropic Raman line profiles and IR line profiles give time-correlation functions of reorientation. Examining them, one founds various correlation times. Theoretical background of such studies is well defined, and a long body of data obtained in Raman and IR experiments is accumulated, see, e.g., ref. [1] and references therein.

It should be mentioned, however, that in the overwhelming majority of the systems analyzed, small molecules in pure liquids and concentrated solutions have been the subject of discussion. This is easy to understand: Fourier transforms are to be made numerically, and in order to perform this operation in the most reliable way, the presence of a single and strong spectral line is needed. Results ob- 
tained for dilute solutions, where spectral lines are weak, and for complex molecules like polymers and biological systems, where spectral lines are often overlapped, are scarce and sometimes unreliable.

The problems arising in Fourier-transform studies are caused by the absence of analytical interrelations between real spectral line profiles and time correlation functions. Only Gaussian and Lorentzian profiles can be Fourier-transformed analytically. However, real band shapes never represent these limiting cases and must be treated numerically.

Similar problem exists in analyses of overlap spectral lines. Usually, these lines are fitted by sums of Gaussians or Lorentzians. In reality, when a composite line is formed by lines of intermediate profiles, such an approach is of no physical meaning. However, the best-known model for intermediate line profiles, the Voigt function, is nonanalytical even in the frequency domain being an integral transform of limiting Gaussian and Lorentzian profiles.

In ref. [2], a novel method has been proposed enabling one to model real line profiles intermediate between Lorentzian and Gaussian by an analytical function which has an analytical counterpart in the time domain. This means that one can find time-correlation functions by fits in the frequency domain, without Fourier transforms, and fit overlapping lines, obtaining dynamic parameters at the same time.

In order to be more specific, let us consider isotropic Raman line profiles. Fourier-transforming them one gets the time-correlation function of vibrational dephasing $G_{V}(t)$ in the following form:

$$
G_{V}(t)=\int_{-\infty}^{+\infty} I_{i s o}(v) \exp (2 \pi i c v t) d t .
$$

Numerous approaches exist to the modeling of $G_{V}(t)$. Generally speaking, the broadening of the isotropic Raman line contour arises due to time-dependent intermolecular interactions which change the instantaneous vibrational frequency $\Delta \omega=f(t)$. Such a process of frequency modulation depends on how the phase memory decays. There are three main types of modulation events, viz., Gaussian-Markovian [3-5], non-Markovian [6,7], and purely discrete Markovian [8-11]. Each of them leads to the correlation functions of vibrational dephasing of different form, which may be generalized through infinite sums [11].

The Gaussian-Markovian, or Kubo case [3-5] remains the most common and widely used in spectroscopic practice. The expression of $G_{V}(t)$ reads as

$$
G_{V}(t)=\exp \left\{-M_{2} \tau_{\omega}^{2}\left[\exp \left(-t / \tau_{\omega}\right)-1+t / \tau_{\omega}\right]\right\}
$$

The method proposed in ref. [2] is based on the model time-correlation function written in the form

$$
G_{V}(t)=\exp \left\{-\left[\left(t^{2}+\tau_{1}^{2}\right)^{1 / 2}-\tau_{1}\right] / \tau_{2}\right\},
$$

whose Fourier transform may be performed analytically giving the vibrational line profile as

$$
I(v)=2 n c \exp \left(\tau_{1} / \tau_{2}\right)\left(\tau_{1}^{2} / \tau_{2}\right) K_{1}(x) / x,
$$

where $x=\tau_{1}\left[4 \pi^{2} c^{2}\left(v-v_{0}\right)^{2}+1 / \tau_{2}^{2}\right]^{1 / 2}, K_{1}(x)$ is the modified Bessel function of the second kind, $v_{0}$ is the peak wavenumber, $n=2$ if $v_{0}=0$, and $n=1$ if $v_{0} \neq 0$. An analysis performed in ref. [2] has shown that eq. 3 explicitly matches eq. 2 .

The approach described in ref. [2] has been carefully tested with single vibrational lines in liquids [12], glasses [13], polymers [14], and ionic melts [15]. As far as overlap lines are concerned, it has been successfully employed in order to obtain vibrational relaxation parameters of isotopic species in liquid carbon tetrachloride from the isotopic structure of the $v_{1}$ line in the isotropic Raman spectrum [16] (see Fig. 1), to characterize instantaneous collision complexes in molten alkali halides [17], and to search for indicators of short-time interactions related to cooperativity in glass-forming liquids [18]. In the next sections, we consider some of these results in more detail. 


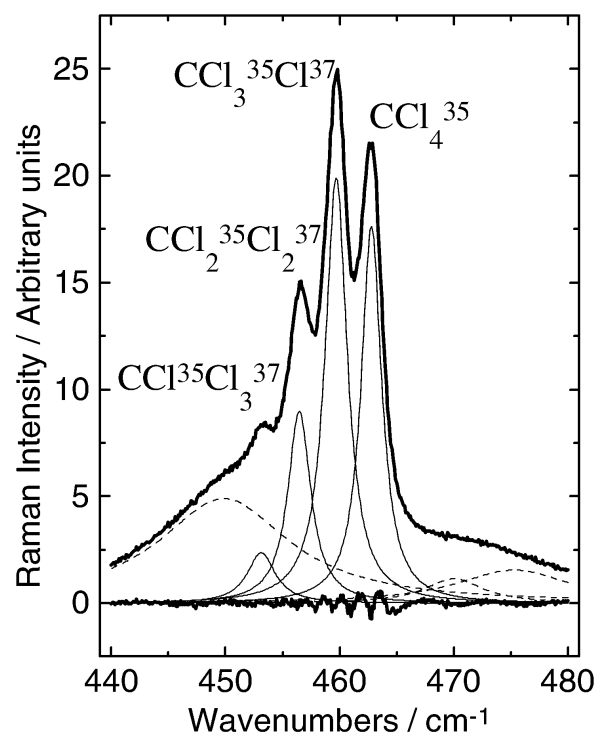

Fig. 1 Isotropic Raman spectrum of $\mathrm{CCl}_{4}$ and its fit. Thick lines: experimental data and residuals. Thin solid lines: isotopic components of the spectrum. Thin dashed lines: binary combinations.

\section{ION-INDUCED DIPOLE ATTRACTION IN MELTS}

It has been mentioned in ref. [1] that the major cause of vibrational dephasing in molecular liquids is Gaussian-Markovian frequency modulation (short phase memory), whereas in ionic liquids, all kinds of modulation processes (from non-Markovian to purely discrete Markovian, from long to completely absent phase memory) are equally possible. In ref. [15], we have presented the first quantitative estimates of the spectroscopically active part of the interaction potential in molten complex chlorides based on dephasing studies. The molten salt systems containing quasi-spherical complex $\mathrm{MCl}_{4}{ }^{2-}$ anions $\left(\mathrm{M}=\mathrm{Mn}^{+2}\right.$ and $\left.\mathrm{Zn}^{+2}\right)$ have been selected, and the study of their $v_{1}\left(\mathrm{~A}_{1}\right)$ isotropic Raman line profiles as a function of the temperature and concentration performed. We have analyzed the form of the timecorrelation function of vibrational dephasing and determined the type of modulation events, which cause the line broadening processes in these systems; these are found to be purely discrete Markovian.

In the theory of purely discrete Markovian modulation elaborated by Burshtein et al. [8-11], the phase memory is considered to be completely absent. The instantaneous frequency shift caused by each collision depends on the distance $r$ to the probe particle and its neighbor as

$$
\Delta \omega=f(r)=C_{k} r^{-k} \text {. }
$$

Equation 5 is crucial in this theory since it makes various types of potentials, which cause modulation, discernible.

The equation enabling one to model time-correlation functions of vibrational dephasing in this case can be written as [11]

$$
-\ln G_{V}(t) / M_{2} \tau_{\omega}^{2}=\frac{3}{k} \sum_{n=0}^{\infty} \frac{(-1)^{n}(t / \tau)^{3 / k+n+1}}{n !(3 / k+n)(3 / k+n+1)} .
$$

$k=3$ corresponds to the Kubo case, and $k=4 \div 12$ signifies a purely discrete Markovian modulation process. Fitting it to the experimental time-correlation functions we have made a judgment about spectroscopically active interactions in complex chlorides.

The best fit of the time-correlation functions of vibrational dephasing in ionic melts containing complex halides of manganese and zinc can be attained at $k=4$ (Fig. 2). This means that the frequency 


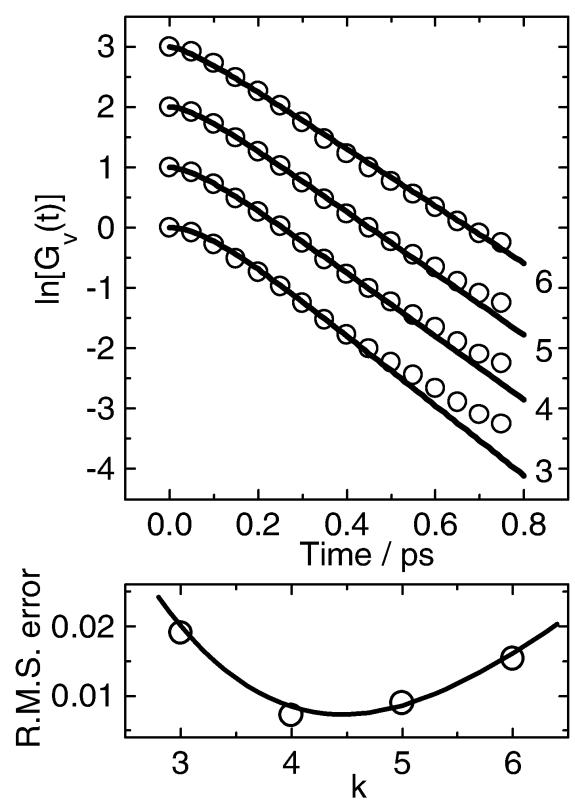

Fig. 2 Time-correlation functions of vibrational dephasing of the totally symmetric vibration of $\mathrm{MnCl}_{4}{ }^{2-}$ (points) and their fits to eq. 6 (lines). Numbers at the curves correspond to different values of $k$ in eqs. 5 and 6 . Upper panel shows the dependence of the r.m.s. error on $k$.

modulation in molten complex chlorides at high temperatures is the purely discrete Markovian process governed by the ion-induced dipole attraction, $k=4$.

\section{DYNAMICS OF MOLTEN ALKALI AND ALKALINE EARTH HALIDES}

Another question to be discussed in this paper touches upon the definition of complex entities in molten salts. More than 40 years ago, Wilmshurst noticed [19] that a mere registration of a vibrational spectrum from molten salts may be ambiguous and, in a general case, cannot be evidence of a complex formation, since the transition time in vibrational spectroscopy, ca. $10^{-2} \mathrm{ps}$, is much less than the lifetime of an instantaneous configuration in an ionic liquid. This means that the vibrational spectrum may reflect the presence of short-lived temporary associates, which have nothing to do with stable complex kinetic entities like $\mathrm{CdCl}_{4}{ }^{2-}, \mathrm{ZnCl}_{4}{ }^{2-}$, etc., existing, say, in crystals or aqueous solutions. This puzzle remains unsolved up until now, since the standard methods of the chemistry of aqueous and nonaqueous solvents enabling one to determine the presence of complex anions in a liquid cannot be applied to molten salts, and only indirect evidence can be used in order to estimate the stability of complexes [20-22].

In ref. [17], the Raman spectra of molten alkali halides have been investigated using the method described in ref. [2]. Parameters of vibrational dephasing have been analyzed, and a dynamic criterion for short-lived complexes has been proposed that can significantly diminish the ambiguity of conclusions regarding the nature of species observable by the methods of vibrational spectroscopy. It is based on a comparison of characteristic times of various processes in molten salts, such as vibrational dephasing times $\tau_{V}$, and frequency modulation times $\tau_{\omega}$, as well as times between collisions $\tau_{B C}$, collision duration times $\tau_{d}$, and the half-period of vibration $T / 2(T=1 / 2 c v$, where $v$ is the vibrational frequency), which plays the role of the damping time of the probe vibrator. Here we give a more exact definition of the short-lived complexes and extend the dynamic criterion to long-lived particles, analyze all existing 
spectroscopic data on complex anions in molten salts, and classify these ions into stable kinetic entities, short-lived complexes, and instantaneous collision complexes. sions.

The analysis of possible relations between $\tau_{V}, \tau_{\omega}, \tau_{B C}, \tau_{d}$, and $T / 2$ leads to the following conclu-

(1) The duration of an act resulting in the vibrational phase shift must be longer than (or at least equal to) the half-period of vibration of the probe oscillator, $\tau_{V} \geq T / 2$. This means that during the $\tau_{V}$ time, when vibrational phase is changing, the particle itself must be able to execute at least one vibration.

(2) The modulation time may vary from this same half-period of vibration $T / 2$ (if the modulation process arises due to interactions of the studied vibrator with a similar neighboring particle) or the time between collisions $\tau_{B C}$ (if collision mechanisms are the motive force of dephasing) to very long times which — in the picosecond time domain — may be considered infinite (structural relaxation, network breakdowns, etc.): $\tau_{\omega} \geq T / 2, \tau_{\omega} \geq \tau_{B C}$.

(3a) If vibrational dephasing and modulation processes elapse during the time equal to the half-period of vibration $T / 2, \tau_{\omega} \approx T / 2, \tau_{V} \approx T / 2$, respective configurations should be considered instantaneous. This means that the bond energy in the configuration in question is very small, and just the first vibration disengages adjacent particles.

(3b) For short-lived complexes, the collision duration time $\tau_{d}$ must be sufficient for a probe particle to execute several vibrations. In this case, the longest of two characteristic times describing the phase decay cannot exceed possible duration of collision, which hence determines the lifetime of a complex: $\tau_{\omega} \leq \tau_{d}, \tau_{V} \leq \tau_{d}$.

In Table 1 the data regarding the $v_{1}\left(\mathrm{~A}_{1}\right)$ totally symmetrical vibrations of $\mathrm{AB}_{4}{ }^{\mathrm{n}-}$ entities of various kinds are presented along with the values of times between collisions and collision duration times. It is clearly seen that for chloride complexes containing $\mathrm{Cs}, \mathrm{Mg}$, and $\mathrm{Ca}$, the frequency modulation time is comparable to the half-period of vibration of the complex entity, $\tau_{\omega} \approx T / 2$, indicating their instantaneous nature.

Table 1 Dynamics of complex species in liquids (all times in ps). The values without references are calculated using experimental data by Bunten et al. [24,25].

\begin{tabular}{|c|c|c|c|c|c|c|c|}
\hline Complex particle & $\mathrm{T} / \mathrm{K}$ & $T / 2$ & $\tau_{B C}$ & $\tau_{\omega}$ & $\tau_{V}$ & $\tau_{d}$ & $\tau_{\omega} / T$ or $\tau_{V} / T$ \\
\hline \multicolumn{8}{|c|}{ Instantaneous species; $\tau_{\omega} \approx T / 2$ or $\tau_{V} \approx T / 2\left(\tau_{\omega} / T \approx 0.5\right.$ or $\left.\tau_{V} / T \approx 0.5\right)$} \\
\hline $\mathrm{CsCl}_{4}^{3-}[5]$ & $623-1123$ & 0.10 & 0.03 & 0.03 & 0.10 & 0.25 & 0.5 \\
\hline $\mathrm{MgCl}_{4}^{2-}$ & 1018 & 0.08 & 0.07 & 0.03 & 0.10 & 1.3 & 0.6 \\
\hline $\mathrm{CaCl}_{4}^{2-}$ & $1073-1173$ & 0.10 & 0.08 & 0.06 & 0.08 & $0.8-1.5$ & 0.4 \\
\hline \multicolumn{8}{|c|}{ Short-lived species; $\tau_{\omega} \leq \tau_{d}$ or $\tau_{V} \leq \tau_{d} ; 0.5 \leq \tau_{\omega} / T \leq 2$ or $0.5 \leq \tau_{V} / T \leq 2$} \\
\hline $\mathrm{LiCl}_{4}^{3-}[5]$ & $623-1123$ & 0.05 & 0.026 & 0.17 & 0.065 & 0.25 & 1.7 \\
\hline $\mathrm{MgBr}_{4}^{2-}$ & 1023 & 0.12 & 0.14 & 0.46 & 0.28 & 1.7 & 1.9 \\
\hline $\mathrm{CaBr}_{4}^{2-}$ & 1043 & 0.16 & 0.15 & 0.044 & 0.19 & 1.0 & 0.6 \\
\hline \multicolumn{8}{|c|}{ Long-lived species; $\tau_{\omega} / T \geq 2$ or $\tau_{V} / T \geq 2$} \\
\hline $\mathrm{MnCl}_{4}{ }^{2-}[23]$ & $818-933$ & 0.061 & 0.11 & 0.12 & 0.25 & - & 2.0 \\
\hline $\mathrm{ZnCl}_{4}^{2-}[23]$ & 873 & 0.066 & 0.09 & 0.06 & 0.31 & - & 2.3 \\
\hline $\mathrm{BiCl}_{4}^{-}[24]$ & $373-573$ & 0.035 & - & 0.78 & 0.41 & - & 11 \\
\hline $\mathrm{CCl}_{4}^{4}[19]$ & 302 & 0.036 & 0.39 & 0.26 & 5.04 & - & 70 \\
\hline
\end{tabular}

Characteristic times of chloride complexes of $\mathrm{Li}$ and bromide complexes of $\mathrm{Mg}$ and $\mathrm{Ca}$ witness the short-lived nature of such entities. Both the modulation and dephasing times of these complexes are 
longer than the half-period of vibration, and the longest of these times does not exceed the collision duration time, $\tau_{\omega} \leq \tau_{d}, \tau_{V} \leq \tau_{d}$.

The data on the dynamics of long-lived species are accessible for the following complex anions in halide melts: $\mathrm{MnCl}_{4}{ }^{2-}$ [15], $\mathrm{ZnCl}_{4}{ }^{2-}$ [15], and $\mathrm{BiCl}_{4}{ }^{-}$[13]. Unfortunately, no data on $\tau_{d}$ have been found, and only indirect evidence can be used regarding the lifetime of these complex particles. Based on electrochemical and thermodynamic data [20-22], we believe that the complexes under discussion could be characterized by a quite long lifetime (at least, of the millisecond order of magnitude) comparable to that of the $\mathrm{CdCl}_{4}{ }^{2-}$ anion measured by Bockris et al. [23]. However, if such a lifetime is ascribed to complexes, the dynamic criterion does not discriminate long-lived and short-lived species: for both types of entities $\tau_{\omega} \leq \tau_{d}, \tau_{V} \leq \tau_{d}$.

Analysis reveals that in order to distinguish between long- and short-lived particles in liquids the ratio between the modulation and dephasing times and the period of vibration can be helpful. As follows from Table 1, for short-lived species the following relations hold: $0.5 \leq \tau_{\omega} / T \leq 2$ or $0.5 \leq \tau_{V} / T \leq$ 2 , whereas for long-lived species $\tau_{\omega} / T \geq 2$ or $\tau_{V} / T \geq 2$. The more stable the complex particle, the greater the $\tau_{\omega} / T$ or $\tau_{V} / T$ ratio, and for the "infinitely" long-lived particle $\left(\mathrm{CCl}_{4}\right)$ it reaches the value of about 70. Therefore, the ratios between the modulation and dephasing times and the period of vibration can serve as the dynamic criterion of long-lived particles in molecular and ionic liquids: $\tau_{\omega} / T \geq 2$ or $\tau_{V} / T \geq 2$.

The results obtained enable one to revisit the autocomplex model by Smirnov, Shabanov, and Khaimenov [26] whose starting point is an a priori assumption that molten alkali halides may be considered as a mixture of autocomplexes $\mathrm{MX}_{4}{ }^{3-}$ and nonbonded cations. Based on this hypothesis the authors proposed detailed procedures for calculating thermodynamic and transport parameters of molten salts and salt mixtures. The autocomplex model is quite popular in numerous applications and enjoys high citation index, in spite of the fact that its authors have never discussed the nature of autocomplexes. The data presented in ref. [17] and this paper may signify that the main idea of the autocomplex model is not far from reality: autocomplexes may be identified as instantaneous and/or short-lived collision complexes.

\section{“ANOMALOUS” FREQUENCY NONCOINCIDENCE EFFECTS}

The so-called frequency noncoincidence effect in liquids manifests itself as splitting of nondegenerate vibrational levels and is considered a powerful tool in studies of interparticle interactions. It is usually treated (see, e.g., refs. $[1,27]$ ) by analogy of Davydov splitting in crystals, i.e. in terms of resonance energy transfer (primarily via transition dipole-transition dipole interactions) between adjacent vibrators; in this case, the frequencies of isotropic Raman lines are higher than the frequencies of anisotropic Raman and IR lines. There are, however, numerous examples of systems showing up the anomalous noncoincidence effects, when the frequencies of isotropic Raman lines appear to be lower than their counterparts in anisotropic Raman and IR.

It has been emphasized in our papers that numerous "anomalies" in vibrational spectra (blue, or negative frequency shifts [28] and anomalous noncoincidence effects [29]) can be treated in terms of repulsion forces, specifically, by means of the derivatives of the vibrational displacement of atoms with respect to the normal coordinate, $d \mathbf{r} / d Q_{k}$. As follows from ref. [1], just blue shifts and anomalous noncoincidence effects are characteristic to molten salts. On the other hand, the analysis of line profiles described in previous sections enables one to carefully determine the peak maxima of vibrational lines and resolve very small non-coincidences. In this section, we examine non-coincidences in liquid benzene and make an attempt to semi-quantitatively discriminate the role of resonant energy transfer and repulsion in frequency noncoincidence effects.

In the simplest case, Davydov-like coupling of a pair of adjacent vibrators leads to the splitting of the vibrational level of the unperturbed vibrator $v_{0}$ to in-phase, low-frequency component $v_{+}$show- 
ing up in the isotropic Raman spectrum and antiphase, high-frequency component $v_{-}$registrable in the anisotropic Raman spectrum and IR absorption:

$$
\begin{array}{ll}
v_{+}=v_{0}+\Delta \omega & \text { (isotropic Raman); } \\
v_{-}=v_{0}-\Delta \omega & \text { (anisotropic Raman, IR). }
\end{array}
$$

Non-coincidences are usually measured as $\Delta v=v_{\text {aniso }}-v_{\text {iso }}=-2 \Delta \omega$, and the positive sign of $\Delta v$ corresponds to negative values of $\Delta \omega$.

According to an exact treatment for crystals [30], the magnitude of $\Delta \omega$ is determined by the derivative of the dipole moment with respect to the normal coordinate, $d \mu / d Q_{k}$, as

$$
\Delta \omega_{k} \propto\left(\chi / M_{k} v_{0 k}\right)\left(d \mu / d Q_{k}\right)^{2},
$$

so that non-coincidences are expected to be greater for the IR-active lines. In eq. $8, \chi$ is the lattice sum and $M_{k}$ is the reduced mass; the $\chi$ value is the same for the vibrators of the same symmetry type.

If vibrational lines are inactive in IR spectra, other resonant energy transfer mechanisms causing noncoincidence effects come into play. If dispersion forces operate between adjacent molecules, noncoincidences are proportional to the values of derivative of the polarizability with respect to the normal coordinate, $d \alpha / d Q_{k}$, and

$$
\Delta \omega_{k} \propto\left(\chi / M_{k} v_{0 k}\right)\left(d \alpha / d Q_{k}\right)^{2} .
$$

The anomalous noncoincidence effect takes place if $\Delta v=v_{\text {aniso }}-v_{\text {iso }}$ appears negative and $\Delta \omega$ is therefore positive. In studies of crystals, it has been first found and interpreted by Lazarev and coworkers [30]. The anomalous noncoincidence effect has a nonresonant nature and depends on kinematic factors. For some vibrations, especially for totally symmetric ones, distances between the atoms of adjacent vibrators vary during in-phase motions and do not change when antiphase motions occur. In this case

$$
\begin{aligned}
& v_{+}=v_{0}+\Delta \omega \quad \text { (isotropic Raman); } \\
& v_{-}=v_{0} \quad \text { (anisotropic Raman, IR), }
\end{aligned}
$$

and the splitting value $\Delta \omega$ depends mainly on repulsion terms. For anomalous noncoincidence effect $\Delta v=v_{\text {aniso }}-v_{\text {iso }}=-\Delta \omega<0$, and the negative sign of $\Delta v$ corresponds to positive values of $\Delta \omega$.

It has been shown [29] that for such Lazarev-like splitting in liquids, the magnitude of $\Delta \omega$ is determined by the derivative of the vibrational displacement $\mathbf{r}$ with respect to the normal coordinate,

$$
\Delta \omega_{k} \propto\left(\kappa \chi / v_{0 k}\right)\left(d \mathbf{r} / d Q_{k}\right)^{2},
$$

where $\kappa$ is the parameter of the repulsion potential, $V(R)=V_{0} \exp (-\kappa R), A$ is a function of the parameters of the repulsion potential and the closest approach distance. Since for totally symmetric vibrations, $d \mathbf{r} / d Q_{k} \propto m^{-1 / 2}$, where $m$ is the mass of the vibrating atom, anomalous non-coincidences should be greater for vibrations involving light atoms.

In the case of benzene, we have studied non-coincidences for two lines of the same $\mathrm{A}_{1 \mathrm{~g}}$ symmetry species: the $v_{1}$ line located at $993 \mathrm{~cm}^{-1}$ and corresponding to $\mathrm{C}-\mathrm{C}$ breathing vibration, and the $v_{2}$ line situated at $3063 \mathrm{~cm}^{-1}$ and corresponding to $\mathrm{C}-\mathrm{H}$ breathing vibration. The Raman spectra of benzene in these regions are shown in Fig. 3; the frequency difference of isotropic and anisotropic components for $v_{1}$ and $v_{2}$ are of different sign: $\Delta v_{1}=-0.26, \Delta v_{2}=+0.60$. This means that the non-coincidences are anomalous for the $v_{1}$ line and "normal" for the $v_{2}$ line. Since the $v_{1}$ and $v_{2}$ lines correspond to the totally symmetric stretching $\mathrm{C}-\mathrm{C}$ and $\mathrm{C}-\mathrm{H}$ modes, Lazarev-like splitting could be expected to play a significant role for both lines. In view of the fact that the $v_{1}$ and $v_{2}$ lines are forbidden in IR, different signs of the non-coincidences may reflect a delicate balance between Lazarev-like (repulsion) effects and Davydov-like splitting caused by resonant energy transfer arising due to induced dipoles. Interestingly, small but distinct anomalous non-coincidences have recently been found for totally sym- 


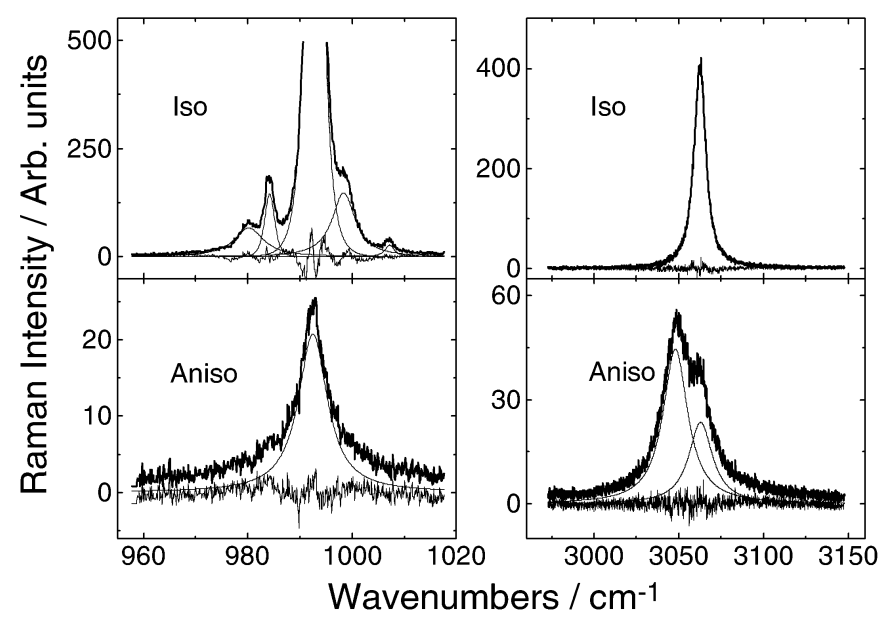

Fig. 3 Raman spectra of benzene in the vicinity of the $v_{1}$ and $v_{2}$ lines and their fits. Thick lines: experimental data and residuals. Thin lines: components of the spectra.

metric $\mathrm{C}-\mathrm{C}$ stretching modes of substituted light and perdeuterated benzenes (toluene, xylenes, and mesithylene) [31].

Practice shows that the results of calculations of the Lazarev splitting are very sensitive to the choice of the parameters of repulsion potential, $\kappa$ and $A$. Therefore, to gain a deeper insight in the relation between Lazarev-like and Davydov-like effects, one can estimate the relative role of each contribution to the overall splitting based on the following equation

$$
\Delta \omega_{k}=\Delta \omega_{D, k}+\Delta \omega_{L, k}=\left(K_{D} / M_{k} v_{0 k}\right)\left(d \alpha / d Q_{k}\right)^{2}+\left(K_{L} / v_{0 k}\right)\left(d \mathbf{r} / d Q_{k}\right)^{2},
$$

where $K_{D}$ and $K_{L}$ are the coefficients determining the weight of Davydov-like and Lazarev-like components, respectively. Here we make a simplification considering that for both vibrations studied, which involve distinctly different atoms (carbon atoms in the case of $v_{1}$ and hydrogen atoms in the case of $v_{2}$ ), the $\kappa$ and $A$ parameters are the same.

Taking $M_{1}=3.32 \cdot 10^{-24} \mathrm{~g}, M_{2}=0.277 \cdot 10^{-24} \mathrm{~g}, v_{01}=993 \mathrm{~cm}^{-1}, v_{02}=3063 \mathrm{~cm}^{-1}$, and using the values of derivatives with respect of the normal coordinates from ref. [32], we get the following system of equations

$$
\begin{aligned}
0.26 & =9.50 \cdot 10^{-18} K_{D}+8.43 \cdot 10^{18} K_{L} & & \text { for } v_{1} ; \\
-0.60 & =2.00 \cdot 10^{-18} K_{D}+3.28 \cdot 10^{18} K_{L} & & \text { for } v_{2} .
\end{aligned}
$$

Finding the values of $K_{D}$ and $K_{L}$ which satisfy this system, we obtain the values of Davydov-like (resonant attraction) and Lazarev-like (kinematic repulsion) contributions to the shift of each vibration studied:

$$
\begin{array}{ll}
\Delta \omega_{D, 1}=-0.09 \mathrm{~cm}^{-1} \text { and } \Delta \omega_{D, 1}=+0.35 \mathrm{~cm}^{-1} & \text { for } v_{1} ; \\
\Delta \omega_{D, 2}=-1.97 \mathrm{~cm}^{-1} \text { and } \Delta \omega_{D, 2}=+1.37 \mathrm{~cm}^{-1} & \text { for } v_{2} .
\end{array}
$$

This means that the nature of the anomalous noncoincidence effect in the case of the $v_{1}$ vibration is almost entirely kinematic. On the other hand, the influence of the resonant induced dipole-induced dipole attraction and kinematic repulsion on the $v_{2}$ mode appears approximately equal, but resonant forces slightly prevail leading to the "normal" non-coincidence. 


\section{CONCLUSIONS}

In this paper, we have tried to demonstrate that the application of the method of vibrational line profile analysis based on the new time-correlation function enables one to elucidate interactions and dynamics in liquids in greater detail than existing approaches. In particular, quantitative description of the interaction potential in molten salts containing halide complexes of manganese and zinc is presented; it is found that the motive force of dephasing processes in these melts are ion-induced dipole forces. Further, dynamics of vibrational dephasing in halide melts is analyzed, and the dynamic criterion of complex entities in a melt is introduced. It is found that the spectroscopic features of molten alkali and alkaline earth halides can be explained in terms of short-lived (or, in some cases, instantaneous) collision complexes.

This same method appears helpful in the studies of the noncoincidence effect in liquids giving an opportunity to carefully determine the peak maxima of vibrational lines and resolve very small non-coincidences. The model treatment of "anomalous" noncoincidence effects in liquids is outlined; it is grounded on the idea of purely kinematic interactions arising due to repulsion forces. We use this treatment for separating the contributions of repulsion and attraction forces resulting in frequency non-coincidences in liquid benzene.

In past, studies of interactions and dynamics in liquids by means of vibrational line profile analyses required the presence of single and strong lines. The approach overviewed in this paper removes this requirement and provides the means for quantitative description of overlapped and weak lines. We hope that this circumstance will significantly extend activities in the field.

\section{REFERENCES}

1. S. A. Kirillov. J. Mol. Liq. 76, 35-95 (1998).

2. S. A. Kirillov. Chem. Phys. Lett. 303, 37 (1999).

3. R. Kubo. In Fluctuations, Relaxation, and Resonance in Magnetic Systems, G. ter Haar (Ed.), p. 27, Plenum Press, New York (1962).

4. M. Constant and R. Fauquembergue. J. Chem. Phys. 58, 4030 (1973).

5. W. G. Rothschild. J. Chem. Phys. 65, 455 (1976).

6. W. G. Rothschild, M. Perrot, F. Guillaume. Chem. Phys. Lett. 128, 591 (1986); J. Chem. Phys. 87, 7293 (1987).

7. S. A. Kirillov. Chem. Phys. Lett. 200, 205 (1992).

8. A. I. Burshtein, S. G. Fedorenko, A. Yu. Pusep. Chem. Phys. Lett. 100, 155 (1983).

9. S. G. Fedorenko, A. Yu. Pusep, A. I. Burshtein. Spectrochim. Acta 43A, 483 (1987).

10. S. A. Kirillov and T. M. Kolomietz. Spectrochim. Acta 48A, 882 (1992).

11. S. A. Kirillov. Chem. Phys. Lett. 202, 459 (1993).

12. S. A. Kirillov and O. Faurskov Nielsen. J. Mol. Struct. 526, 317 (2000).

13. S. A. Kirillov and S. N. Yannopoulos. Phys. Rev. B 61, 11391 (2000).

14. S. A. Kirillov and T. M. Kolomiyets. J. Phys. Chem. B 105, 3168 (2001).

15. S. A. Kirillov, G. A. Voyiatzis, I. S. Musiyenko, G. M. Photiadis, E. A. Pavlatou. J. Chem. Phys. 114, 3683 (2001).

16. S. A. Kirillov. J. Raman Spectrosc. 33, 195 (2002).

17. S. A. Kirillov, G. N. Papatheodorou, E. A. Pavlatou. J. Chem. Phys. 116, 9341 (2002).

18. S. A. Kirillov and S. N. Yannopoulos. J. Chem. Phys. 117, 1220 (2002).

19. J. K. Wilmshurst. J. Chem. Phys. 39, 1779 (1963).

20. A. D. Pelton. Can. J. Chem. 49, 3919 (1971).

21. A. S. Kucharsky and S. N. Flengas. J. Electrochem. Soc. 119, 1170 (1972).

22. B. F. Markov. Thermodynamics of Complex Compounds in Molten Salt Systems, Naukova Dumka, Kiev (1988). 
23. J. O’M. Bockris, D. Inman, A. K. N. Reddy, S. Srinivasan. J. Electroanal. Chem. 5, 476 (1963).

24. R. A. J. Bunten, R. L. McGreevy, E. W. J. Mitchell, C. Raptis, P. J. Walker. J. Phys. C: Solid State Phys. 17, 4705 (1984).

25. R. A. J. Bunten, R. L. McGreevy, E. W. J. Mitchell, C. Raptis. J. Phys. C: Solid State Phys. 19, 2925 (1986).

26. M. V. Smirnov, O. M. Shabanov, A. P. Khaimenov. Elektrokhimija 2, 1240 (1966).

27. A. Morresi, M. Paolantoni, P. Sassi. Recent Res. and Devel. in Chem. Phys. 1, 67 (2000).

28. S. A. Kirillov. Spectrochim. Acta 48A, 861 (1992).

29. S. A. Kirillov. J. Raman Spectrosc. 24, 167 (1993).

30. A. N. Lazarev, A. P. Mirgorodskii, M. B. Smirnov. Vibrational Spectra and Dynamics of IonicCovalent Crystals, Nauka, Leningrad (1985); Solid State Commun. 58, 371 (1986).

31. A. Morresi, M. Paolantoni, P. Sassi, R. S. Catagliotti, G. Paliani. J. Phys.: Condens. Matter 12, $3631(2000)$.

32. J. M. Férnandez-Sánchez and S. Montero. J. Chem. Phys. 90, 2909 (1989). 